12

\title{
О способе увеличения предельно допустимого уровня ударных токов короткого замыкания в электрических контактах
}

\author{
() А.М. Чалый, В.А. Дмитриев, М.А. Павлейно, О.М. Павлейно, М.С. Сафонов
}

Санкт-Петербургский государственный университет, физический фракультет, 198504 Петергоф, Санкт-Петербург, Россия

e-mail: pavleyno@mail.ru

(Поступило в Редакцию 19 июля 2018 г.)

\begin{abstract}
При протекании тока через электрические контакты происходит дополнительное тепловыделение, связанное с наличием контактного сопротивления. Это может привести к их значительному нагреву. В сильноточных контактах высоковольтных электрических аппаратов проблема нагрева проявляется наиболее остро при протекании аварийных токов короткого замыкания. Максимально допустимый уровень таких токов ограничен нагревом контактных областей до температуры плавления. Сварки, вызванные плавлением материала контактов, как правило, приводят к потере работоспособности аппаратов. Предложен способ значительного увеличения уровня допустимых токов короткого замыкания. Он состоит в предварительном последовательном импульсном нагреве контактов до температуры, превышающей температуру рекристаллизации материала. Этот эффект подтвержден экспериментально. Проведено численное моделирование процесса импульсного нагрева. Его результаты позволили выработать рекомендации по выбору параметров серии импульсов тока, воздействие которых на контакты позволяет заметно увеличить их стойкость к токам короткого замыкания.
\end{abstract}

DOI: $10.21883 / J T F .2019 .04 .47322 .286-18$

\section{Введение}

Электрические контакты высоковольтного сетевого оборудования в процессе эксплуатации периодически подвергаются токовым воздействиям, величина которого может значительно превосходить уровень номинальных токов. Это может происходить как в штатном режиме, например, при включении или отключении мощных электродвигателей, конденсаторных батарей значительной емкости, так и при возникновении коротких замыканий в линиях электропередачи.

Наибольшую опасность с точки зрения возможной потери работоспособности оборудования представляют токи короткого замыкания. Типичный вид осциллограммы тока при возникновении короткого замыкания приведен на рис. 1.

До аварии через контакты, как правило, протекает номинальный ток $I_{r}$. В результате возникновения короткого замыкания ток возрастает до величины $I_{s-c}$ (установившееся значение тока короткого замыкания), которая определяется параметрами сети и параметрами аварии и может более чем на порядок превосходить номинальный ток. Предельные наиболее критичные для контактов параметры токов короткого замыкания и их форма определены в [1].

При классификации токовых воздействий на электрические контакты особо выделяют начальный участок короткого замыкания, на котором возможен значительный бросок тока, величина которого зависит от ряда факторов, в частности, от момента (фазы) возникновения короткого замыкания и параметров электрической сети [1]. Величина тока в первом полупериоде, называ- емая ударным током $I_{s h}$, может практически в два раза превосходить установившееся значение тока короткого замыкания $I_{s-c}$. Именно ударный ток короткого замыкания может стать причиной нагрева контактной области до плавления и возникновения фатальной сварки, приводящей к потере работоспособности коммутационного оборудования.

Предельно допустимый уровень ударного тока короткого замыкания $I_{s h}^{\mathrm{lim}}$ является важной характеристикой электрических аппаратов, в значительной степени определяющий их эксплуатационные характеристики. В [2] показано, что для сильноточных контактов под $I_{s h}^{\lim }$ следует понимать минимальный ток начала плавления контактных пятен $I_{m e l t}^{\min }$, так как даже при незначительном превышении величиной ударного тока этого значения

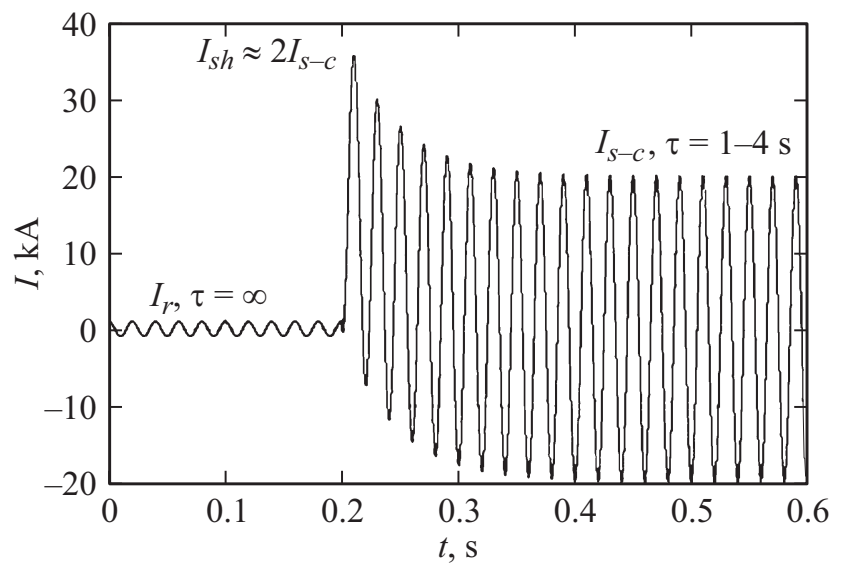

Рис. 1. Типичный вид осциллограммы тока при возникновении короткого замыкания. 

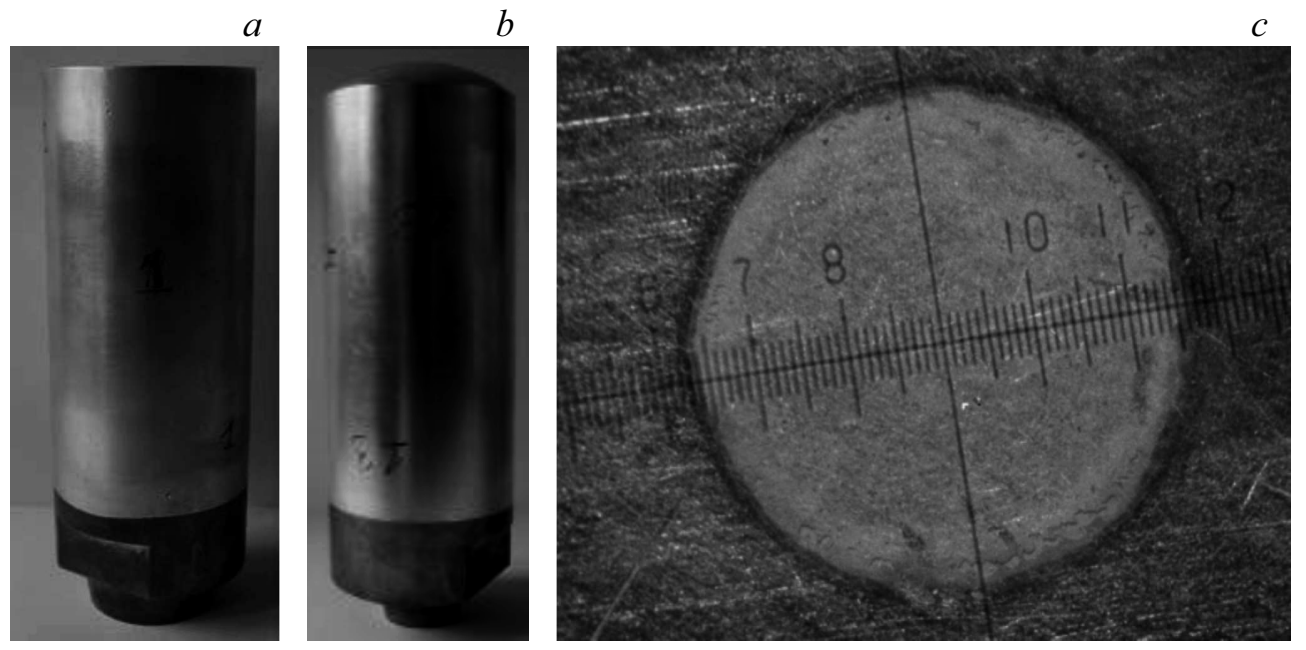

Рис. 2. Исследуемые контакты: с плоской $(a)$ и сферической $(b)$ контактными поверхностями, микрофотография контактного пятна $(c)$.

наблюдается резкий рост силы отрыва сварки. В свою очередь, величина $I_{m e l t}^{\min }$ определяется величиной контактного сопротивления.

Увеличить $I_{s h}^{\lim }$ можно путем оптимизации конструкции контактов, учитывая, что контактное сопротивление зависит от размера и формы контактных пятен, от формы границ контактов и других геометрических параметров [3-5]. Другим способом увеличения $I_{s h}^{\lim }$ является повышение значения силы контактного нажатия $F_{\text {cont }}$, что снижает величину контактного сопротивления. Однако такой способ не всегда применим. Это связано в том числе и с тем, что увеличение силы, как правило, имеет своим следствием увеличение габаритов коммутационных аппаратов и снижение их быстродействия. В ряде случаев максимальное значение силы ограничено требованиями к условиям эксплуатации, например, если коммутация осуществляется в ручном режиме.

Ниже будет показано, как можно существенно увеличить уровень предельно допустимого ударного тока без изменения конфигурации контактов и без увеличения силы контактного нажатия. Предлагаемый способ заключается в предварительной термической обработке окрестности контактных пятен путем пропускания через контакты серии импульсов ударного тока с постепенно увеличивающейся амплитудой при неизменной силе контактного нажатия.

\section{Эксперимент}

Было проведено исследование прохождения ударных токов короткого замыкания через замкнутые электрические контакты, представляющие собой соосные цилиндрические электроды, соприкасающиеся своими торцевыми поверхностями. Торец одного электрода имеет плоскую поверхность, торец другого представляет собой сегмент сферы (рис. 2, $a, b)$. Это обеспечивает при

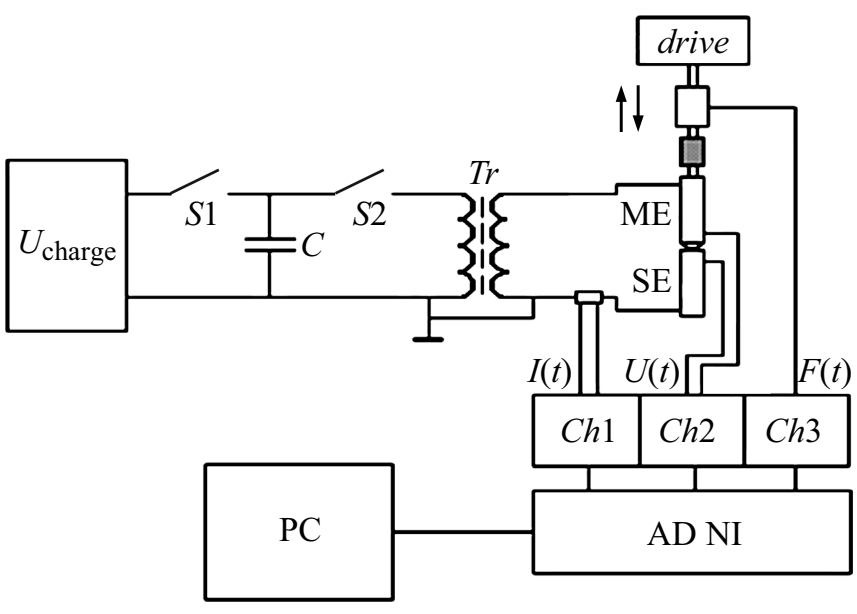

Рис. 3. Блок-схема экспериментальной установки.

приложении внешней силы формирование контактного пятна, близкого по форме к кругу (рис. 2,c).

Блок-схема экспериментальной установки для исследования стойкости контактов к ударному току приведена на рис. 3.

Испытательный ток формируется ударным контуром, состоящим из батареи конденсаторов $C$ (160 параллельно соединенных конденсаторов емкостью по $50 \mu \mathrm{F})$ и первичной обмотки ударного трансформатора $T r$. Коммутаторы $S_{1}$ и $S_{2}$, представляющие собой высоковольтные вакуумные выключатели, в исходном положении находятся в отключенном состоянии. При замыкании $S_{1}$ происходит заряд батареи конденсаторов от источника $U_{\text {charge. }}$ Максимальное значение напряжения составляет $8 \mathrm{kV}$.

После отключения $S_{1}$ и включения $S_{2}$ батарея разряжается через первичную обмотку понижающего трансформатора. При этом через замкнутые испытуемые 

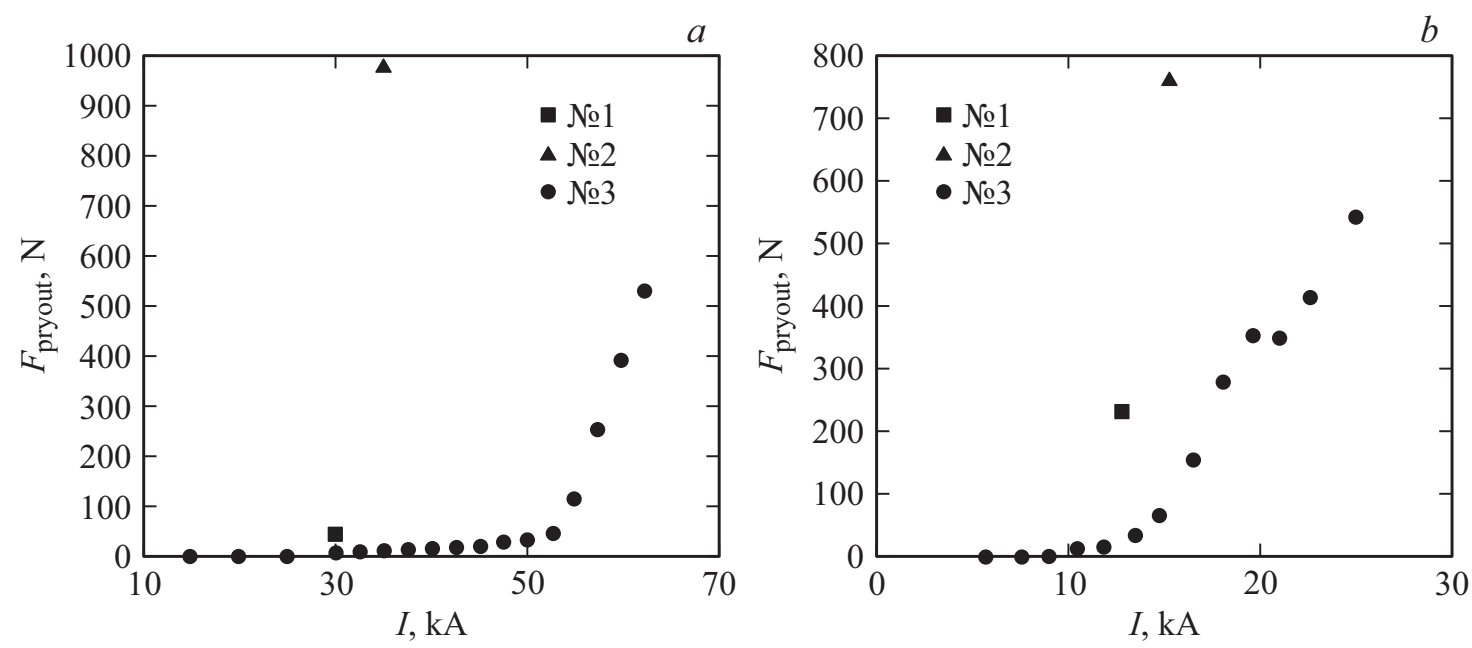

Рис. 4. Зависимость силы отрыва контактов от величины ударного тока при $F_{\text {cont }}=2500(a)$ и $F_{\text {cont }}=300 \mathrm{~N}(b)$.

контакты (неподвижный и подвижный электроды - SE и $\mathrm{ME}$ ), включенные во вторичную обмотку, протекает затухающий квазипериодический ток. Значение ударного тока определяется начальным напряжением заряда батареи конденсаторов, а частота - величиной емкости $C$ и параметрами трансформатора. Величина тока в первом полупериоде может достигать $100 \mathrm{kA}$.

Электроды приводятся в механическое взаимодействие с помощью привода на основе пневмоцилиндра. Сила контактного нажатия может варьировать от 0 до $3000 \mathrm{~N}$.

Установка содержит три измерительных канала $C h 1$, $C h 2$ и Ch3 для записи осциллограмм протекающего тока, напряжения на электродах и показаний с тензометрического датчика усилий для регистрации силы отрыва контактов при возникновении сварки.

В [6] показано, что сварка медных контактов ударными токами начинается при нагреве окрестности контактных пятен до температуры около $700 \mathrm{~K}$. На этом этапе она носит диффузионный характер. Такие сварки характеризуются гораздо меньшими силами отрыва контактов, чем сварки, вызванные плавлением, и, как правило, не являются фатальными с точки зрения потери функциональности аппаратов.

Определить сварка какого типа возникла при протекании ударного тока можно по анализу поверхности контактных пятен после разрыва контактов или по виду осциллограммы напряжения. В момент начала плавления на осциллограмме возникает характерная особенность, вызванная изменением контактного сопротивления при фазовом переходе [2], что позволяет идентифицировать этот тип сварки.

Было проведено сравнение результатов воздействия на контакты ударных токов в двух режимах. В первом случае через контакты пропускался один импульс тока и измерялась сила отрыва сварки. После этого контакты извлекались из установки, проводилось исследование контактной поверхности на предмет определения типа сварки. Во втором случае через контакты пропускалась серия импульсов ударного тока с измерением силы отрыва контактов после каждого импульса. Исследование поверхности проводилось после воздействия всей серии импульсов.

Было обнаружено, что при определенных условиях результаты прохождения тока одной и той же величины через идентичные по форме контакты и при одинаковой силе контактного нажатия могут быть принципиально разными в этих режимах. Покажем это на конкретном примере.

Использовались три одинаковые пары цилиндрических электродов при силе контактного нажатия $2500 \mathrm{~N}$. Через две из них были пропущены одиночные импульсы тока, величина которого была подобрана таким образом, чтобы в одном случае сварка была незначительной по силе (пара электродов № 1), а в другом случае было зафиксировано начало плавления (пара № 2). Через третью пару контактов была пропущена последовательность импульсов тока возрастающей амплитуды, начиная с величины $15 \mathrm{kA}$. Назовем такой режим „тренировкой контактов. На рис. 4, $a$ представлена зависимость силы отрыва контактов $F_{\text {pryout }}$ от величины протекающего тока.

При однократном пропускании ударного тока величиной $30 \mathrm{kA}$ через первую пару контактов было зафиксировано возникновение диффузионной сварки с силой отрыва $50 \mathrm{~N}$. Для второй пары при токе $35 \mathrm{kA}$ сила отрыва была гораздо больше - $980 \mathrm{~N}$, и при этом на осциллограмме напряжения присутствовала особенность, характерная для плавления. Отметим довольно малое различие по току между начальной диффузионной сваркой и сваркой плавлением - всего $5 \mathrm{kA}$.

Для третьей пары электродов наблюдался весьма интересный, особенно с количественной точки зрения, эффект. Сварка, начавшись при токе $30 \mathrm{kA}$, практически не изменяла свою интенсивность в очень широком диапазоне токов, все время оставаясь на начальном 
уровне сваривания контактов. Лишь при увеличении тока до $52.5 \mathrm{kA}$ сила отрыва достигла той же величины $(50 \mathrm{~N})$, что и при однократном прохождении тока $30 \mathrm{kA}$ через первую пару контактов, при том, что разница по току между этими двумя случаями очень велика более $20 \mathrm{kA}$.

В результате последовательного прохождения импульсов тока также значительно изменился и уровень минимального тока плавления. Для пары электродов № 2 он был равен $35 \mathrm{kA}$. Для третьей пары плавления не наблюдалось даже при токе $62.5 \mathrm{kA}$. Судя по зависимости силы отрыва сварки от тока, значение минимального тока плавления контактов в режиме многократного прохождения тока возросло до значения, большего чем $65 \mathrm{kA}$. А именно величина предельно допустимого ударного тока увеличилась приблизительно на $30 \mathrm{kA}$ (около 90\%).

Аналогичные результаты были получены и для других значений силы контактного нажатия, которая варьировала в широком диапазоне - от 300 до 2500 N. Данные по сварке контактов для нижней границы рассмотренного диапазона сил приведены на рис. $4, b$. Эффект увеличения $I_{s h}^{\lim }$ в результате тренировки контактов здесь также явно выражен: величина предельно допустимого уровня ударного тока выросла примерно на $10 \mathrm{kA}$ (более 60\%).

Отметим, что во всех проведенных экспериментах при варьировании силы контактного нажатия в указанном широком диапазоне значений в результате тренировки контактов наблюдалось увеличение $I_{s h}^{\mathrm{lim}}$ более чем на $50 \%$.

\section{Обсуждение экспериментальных данных}

Обнаруженный эффект существенного увеличения предельно допустимого уровня ударного тока (тока начала плавления) в результате последовательного импульсного токового воздействия увеличивающейся амплитуды на контакты связан с необратимыми изменениями, происходящими в окрестности контактных пятен при последовательном пропускании импульсов тока [7].

При приложении к контактам внешней силы $F_{\text {cont }}$ происходит деформация контактной области и формирование площадки смятия, образующей контактное пятно. Если величина $F_{\text {cont }}$ достаточно велика, что характерно для сильноточных контактов, то наряду с упругими деформациями присутствуют также и области, деформированные пластически.

При пропускании тока происходит локальный нагрев окрестности контактных пятен, механические свойства материала при этом изменяются. Это приводит к тому, что зона необратимых пластических деформаций расширяется. Это будет особенно заметно, если нагрев произошел до температуры, больше чем температура размягчения (рекристаллизации) материала. После остывания радиус контактного пятна оказывается больше, чем до пропускания тока, а величина контактного сопротивления уменьшается. Поэтому при прохождении следующего импульса тока, несколько большего по величине, чем предыдущий, температура контактной области может заметно не возрасти, и увеличение силы отрыва сварки может быть незначительным.

При правильном выборе шага приращения тока будет происходить последовательное накопление пластических деформаций, вызывающее постепенное увеличение радиуса контактного пятна от импульса к импульсу. Это и вызывает смещение границы тока начала плавления при неизменной величине силы контактного нажатия.

Подтвердим справедливость данных рассуждений численными расчетами для первого из рассмотренных выше случаев (рис. $4, a)$.

Будем использовать методику расчета импульсного нагрева контактов, описанную в работах [2,6]. Расчеты проводятся в следующей последовательности. По измеренной до пропускания тока величине контактного сопротивления рассчитывается начальное значение радиуса контактного пятна. Далее решается задача нестационарного нагрева электродов током известной величины $I(t)$. Решение проводится итерационно: на каждом шаге по времени задается ток и подбирается такое значение радиуса контактного пятна $a$, которое обеспечивает достаточную близость расчетного значения напряжения на электродах к экспериментально измеренному. То есть подбором функции $a(t)$ мы приближаем расчетную кривую $U_{c}(t)$ к реальной осциллограмме напряжения. Учитывается зависимость от температуры теплоемкости, теплопроводности, плотности и удельного сопротивления материала. При этом в каждый момент времени мы знаем распределение температуры и потенциала в контактной области, а следовательно, и распределение тепловых потоков, напряженности электрического поля, плотности тока и ряда других величин.

Для всех реализаций пропускания тока были проведены численные расчеты, в результате чего получена информация о нагреве контактной области и об изменении размеров контактного пятна. На рис. 5 приведены данные о величине радиуса контактного пятна перед пропусканием каждого импульса тока и значение максимальной температуры контактного пятна в процессе нагрева каждым импульсом тока.

Исходный радиус контактного пятна был практически одинаковым для трех пар электродов - около $1 \mathrm{~mm}$. Прохождение тока $30 \mathrm{kA}$ для электродов № 1 вызвало нагрев контактной области $1070 \mathrm{~K}$, что значительно ниже температуры плавления меди, которая равна 1356 K. Такой нагрев вызывал сварку с небольшой силой отрыва в $50 \mathrm{~N}$. При протекании тока большей амплитуды (35 kA) через вторую пару электродов нагрев увеличился до температуры плавления. Это привело к значительному росту силы отрыва электродов до $1000 \mathrm{~N}$.

Проанализируем теперь прохождение последовательности импульсов тока через третью пару электродов. Процесс тренировки контактов был начат с низкого 

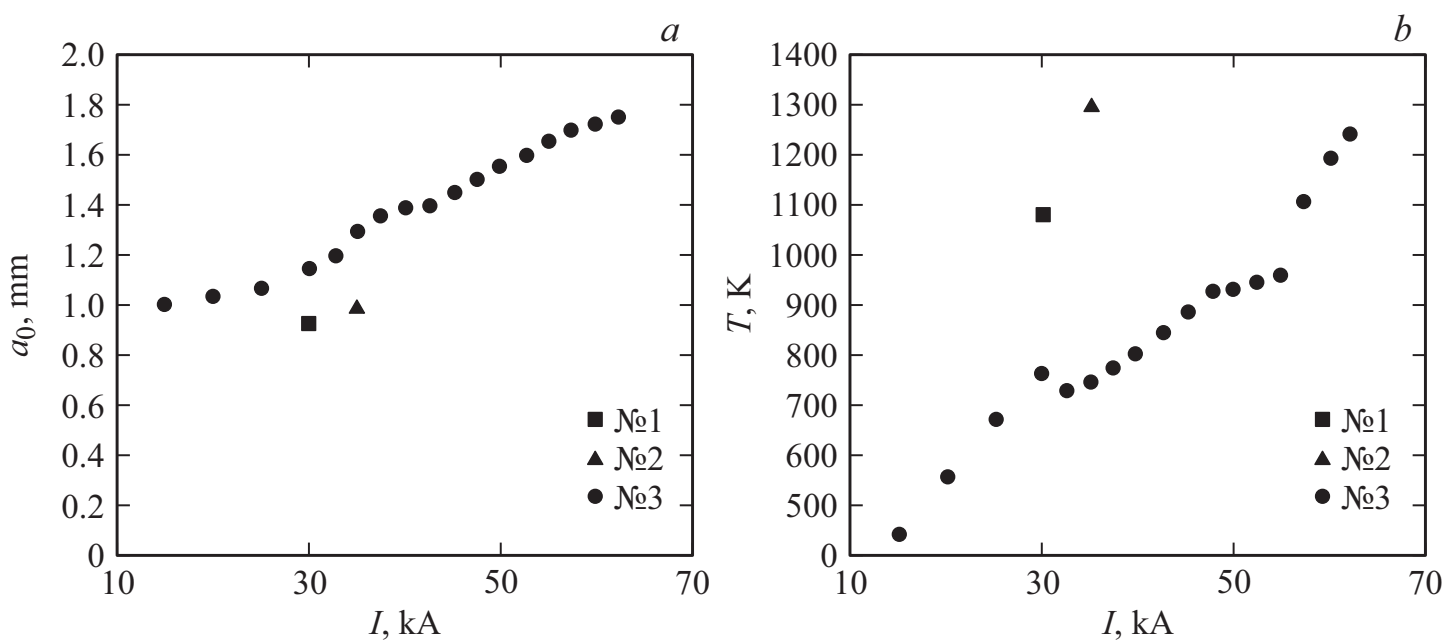

Рис. 5. Изменение радиуса контактного пятна $(a)$ и максимальной температуры нагрева $(b)$ в процессе „тренировки“ контактов.

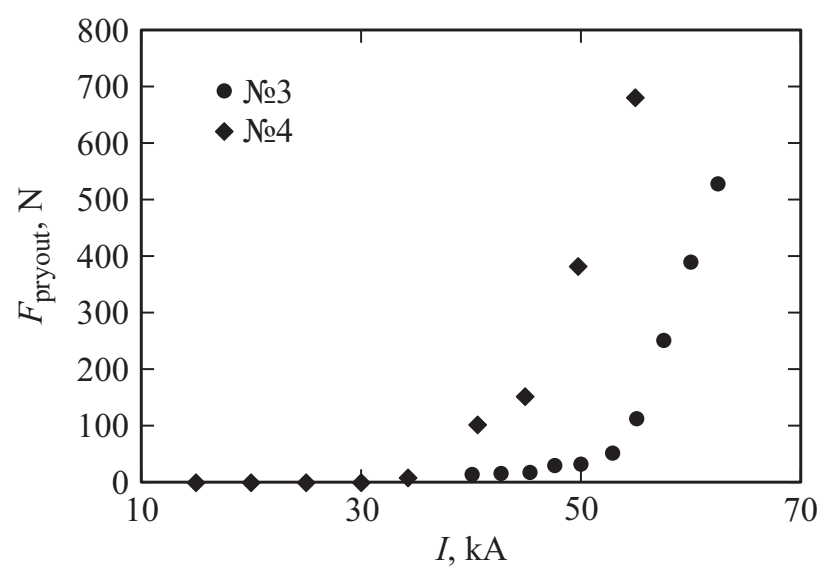

Рис. 6. Зависимость силы отрыва контактов от тока при разных значениях приращения тока.

уровня тока $15 \mathrm{kA}$, когда сварки контактов еще не происходит.

В результате прохождения первого импульса тока контактная область нагрелась до температуры, меньшей температуры размягчения меди $T_{\text {soft. }}$ Вследствие этого увеличение радиуса было небольшим, около 5\%. Приращение тока было выбрано равным $5 \mathrm{kA}$. Два последующие импульса тока нагревали контактную область до температуры выше $T_{\text {soft }}$, поэтому имело место большее расплывание пятна. При токе $30 \mathrm{kA}$ была достигнута температура сваривания контактов $T_{\text {weld}}$, сила отрыва составила $8 \mathrm{~N}$.

Для того чтобы при следующем импульсе температура существенно не превысила $T_{\text {weld }}$ (что привело бы к заметному возрастанию интенсивности сварки и, по сути, к вынужденному прекращению процесса тренировки), шаг приращения тока был уменьшен вдвое до $2.5 \mathrm{kA}$. В результате этого два следующие импульса нагревали контактную область до меньшей температуры, чем предыдущий. Поэтому и сила отрыва сварки при этом практически не возросла.

Ситуация, когда температура нагрева не существенно превышала $T_{\text {weld }}$, сохранилась в довольно широком диапазоне токов до уровня $55 \mathrm{kA}$. После этого температура контактного пятна и соответственно интенсивность сварки начала быстро увеличиваться от импульса к импульсу и при токе $62.5 \mathrm{kA}$ составила $530 \mathrm{~N}$.

Смещение границы тока начала плавления, связанное с тренировкой контактов, существенно зависит от шага приращения тока. Чтобы показать это, проанализируем результаты, полученные для еще одной пары электродов такой же формы (№ 4) при той же силе контактного нажатия $2500 \mathrm{~N}$ (рис. 6). Здесь приращение тока после начала сваривания контактов не уменьшалось, как в рассмотренном случае, до $2.5 \mathrm{kA}$, а оставалось равным $5 \mathrm{kA}$.

При приращении тока между импульсами величиной $2.5 \mathrm{kA}$ температура контактного пятна, приводящая к сварке с силой отрыва $400 \mathrm{~N}$, была достигнута при значении тока $60 \mathrm{kA}$, при увеличении шага в два раза до $5 \mathrm{kA}$ соответствующее значение тока снизилось до $50 \mathrm{kA}$. То есть эффект от тренировки при таком выборе режима токовых воздействий снизился примерно на $10 \mathrm{kA}$ (см. рис. 6, на нем для пары электродов № 3 приведен фрагмент зависимости $\left.F_{\text {pryout }}(I)\right)$.

\section{Заключение}

В заключение остановимся на некоторых обстоятельствах, которые следует учитывать при выборе режима тренировки контактов. Первое, что стоит отметить, это отсутствие однозначного решения задачи о поиске оптимальной последовательности токовых воздействий. Это связано с отсутствием возможности аналитического решения задачи об импульсном нагреве контактов или ее численного решения в замкнутой постановке, без необходимости привлечения экспериментально полученных 
данных. При наличии такой возможности можно было бы поставить задачу поиска оптимальной, с точки зрения максимального увеличения значения тока плавления контактов, последовательности импульсов тока $I_{k}(t)$, $k=1, \ldots, K$ при фиксированном числе импульсов $K$.

Однако с учетом полученных результатов можно сформулировать ряд положений, которые окажутся полезными при выборе режима тренировки контактов. Величину первого импульса тока следует выбирать меньше, чем ток начала сварки. По-видимому, оптимальное начальное значение тока соответствует нагреву контактной области до температуры, равной или несколько меньшей температуры размягчения материала. Приращение величины тока должно быть таким, чтобы после очередного импульса тока температура контактной области не достигла величины, при которой сила отрыва сварки значительно превосходит уровень, характерный для начального этапа сваривания контактов. Уменьшение приращения тока между импульсами приводит к усилению эффекта тренировки, однако оно приводит к увеличению длительности процедуры, так как число импульсов возрастает.

Несмотря на то, что рекомендации по выбору режима тренировки контактов не носят четко выраженный количественный характер (что едва ли возможно в общем случае), их знание позволит эффективно подобрать режим для конкретного типа контактов при проведении минимального числа измерений.

Экспериментально показана возможность значительного увеличения (более 50\%) предельно допустимого уровня ударного тока через электрические контакты без изменения их геометрических параметров и силы контактного нажатия путем предварительной „тренировки“ контактов - пропускания через них импульсов тока с возрастающей амплитудой.

Причиной проявления данного эффекта является накопление пластических деформаций в контактной области вследствие многократного нагрева до температуры выше температуры размягчения материала, что приводит к последовательному росту радиуса контактного пятна и, следовательно, к уменьшению величины контактного сопротивления.

\section{Список литературы}

[1] ГОСТ 52735-2007 Короткие замыкания в электроустановках. Методы расчета в электроустановках переменного тока напряжением свыше 1 кВ. М.: Стандартинформ, 2007. $35 \mathrm{c}$.

[2] Автореф. канд. дис. Павлейно О.М. Физические особенности нагрева сильноточных электрических контактов: 01.04.13. СПб. 2015. 148 с.

[3] Хольм Р. Электрические контакты. М.: ИЛ, 1961. 464 с.

[4] Slade P.G. Electrical contacts: principles and applications. NY: CRC Press, 2013. 1311 p.
[5] Braunovic M., Konchits V.V., Myshkin N.K. Electrical Contacts: Fundamentals, Applications and Technology. NY.: CRC Press, 2006. $672 \mathrm{p}$.

[6] Chalyi A.M., Dmitriev V.A., Pavleino M.A., Pavleino O.M. // Surf. Eng. Appl. Elect. 2013. Vol. 49. N 5. P. 433-439.

[7] Павлейно О.М., Павлейно М.А., Сафонов М.С. // Сб. докл. XI Всерос. съезда по фундаментальным проблемам теоретической и прикладной механики. Казань, 2015. С. $2887-$ 2889. 